\title{
QoS Guaranteed Vertical Handoff Signaling Scheme for Heterogeneous Networks ${ }^{1}$
}

\author{
WANG Yihong ${ }^{1}$, ZHANG Qi ${ }^{1}$, WANG Ying ${ }^{1}$, ZHANG Ping ${ }^{1}$, Victor O.K. Li $^{2}$ \\ (1. WTI Labs, Beijing University of Posts and Telecommunications, P.O.BOX 92 , Beijing 100876, China; \\ wyh bupt@163.com \\ 2. Multimedia Networking Lab. Department of Electrical and Electronic Engineering University of Hong Kong, Hong \\ Kong, China )
}

\begin{abstract}
Signaling process of vertical handoff with QoS support between GPRS and WLAN is developed. Schemes of Handoff Preparing Loop (HPL) and Layer 3 (Mobile IP layer) bi-direction comnections between GPRS and WLAN are proposed in order to provide QoS guarantee. However, bi-direction connection will occupy more network resources. To solve this problem, a parameter "connecting lifetime" is introduced. The latency analysis and simulation results show that systems could achieve better performance in terms of handoff latency and outage probability.
\end{abstract}

\section{INTRODUCTION}

With the development of wireless telecommunication industry, the needs of mobile users for wide bandwidth services as well as large-scale access availability, such as real-time multimedia service, are increasing. However, most existing wireless networks can be divided into two categories: those that provide low bandwidth services over a wide geographic area, and those that provide high bandwidth services over a limited geographic area. It is necessary to find a way to integrate both kinds of networks to provide high bandwidth and large-scale coverage. In particular, WLAN (such as IEEE 802.11b) provides available $11 \mathrm{Mbps}$ service in hotspots. Cellular wireless networks can cover large-scale areas, while providing limited bandwidth, e.g. GPRS network provides up to 171.2 Kbps data rate[1]. It is desirable to use WLAN network in hotspot as much as possible to enjoy high data rate, low power consumption and low charge; when away from hotspots, handoff to GPRS network is made to maintain ongoing communications. Therefore seamless vertical handoff between heterogeneous networks is inevitable.

Implementation of vertical handoff with QoS guarantee is one of key problems. [2][3]propose a vertical handoff

\footnotetext{
${ }^{1}$ This research is supported by the NSFC/RGC Joint Research Scheme. Project No.
}

decision algorithm based on fuzzy logic. This paper mainly studies signaling process between WLAN and GPRS, and proposes a scheme of QoS guaranteed handoff signaling process.

Mobility management solutions in heterogeneous networks consist of three categories: Layer 3 solution, Layer 2 solution and Cross-layer solution [4]. The proposed scheme belongs to the third category.

The rest of the paper is organized as follows. Section II introduces structure of integrated networks. Section III describes signaling process scheme. In section $N$, detailed handoff signaling process flow is presented. And analysis for the latency of L3 handoff is given. Simulation and analysis is given in section V. Conclusion is drawn in section VI.

\section{INTEGRATION ARCHITECTURE OF WLAN AND GPRS}

Two kinds of architectures are popular for WLANGPRS combination: tight coupling and loose coupling. [5]describes the tight coupling and loose coupling structures. With the method of tight coupling, WLAN is usually considered as one of the GPRS cells, which leads to simple structure, but the low speed bottleneck cannot be overcome for the core network is still the GPRS network. The IETF Mobile IP based structure belongs to loose coupling architecture, but it is not described in detail in [5]. We propose the structure based on Mobile IP. The core network is Mobile IP network. GPRS and WLAN networks are fully overlapped. GPRS network is the home network, attached to Home Agent (HA), and all the WLAN networks are visiting networks. When Mobile Station (MS) moves into the hotspot of WLAN, MS obtains a Foreign Agent Care-of Address (CoA), which means that the data is encapsulated at $\mathrm{HA}$, tunneled to FA, and decapsulated at $\mathrm{FA}$, then delivered to MS.

\section{SCHEME OF VERTICAL HANDOFF SIGNALING}

Vertical handoff of heterogeneous networks is usually complicated and of large latency. In order to provide QoS 
guaranteed handoff, the Handoff Preparing Loop (HPL) is introduced. HPL is the area near the edge of WLAN hotspot, where handoff is about to perform. It is designed that all the handoff signaling exchanges in HPL. And before Layer 2 disconnection with the old network, the new Layer 3 connection is set up in HPL, namely bidirection connection. Then Layer 2 handoff occurs. HPL could provide more time for systems to prepare for vertical handoff, leading to low handoff latency and low outage probability.

\section{A. Handoff Preparing Loop}

When MS moves from GPRS to WLAN, it is defined that the spot where the first signal from WLAN above threshold is received is the start point of HPL, and handoff spot is the end point of HPL. When MS moves to GPRS from WLAN, the spot where the first signal from WLAN below threshold is received is the start point, and the handoff spot is the end point. As shown in figure 1 and 2.

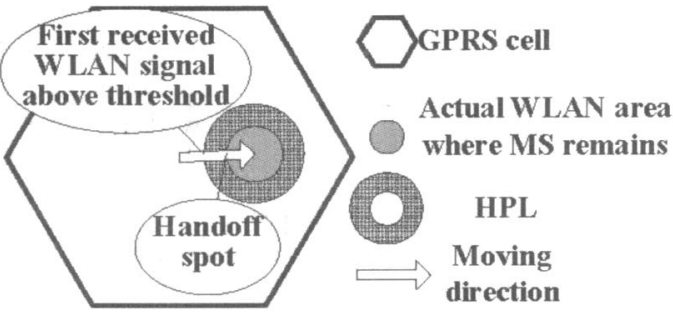

figure $1 \mathrm{HPL}$ : from GPRS to WLAN

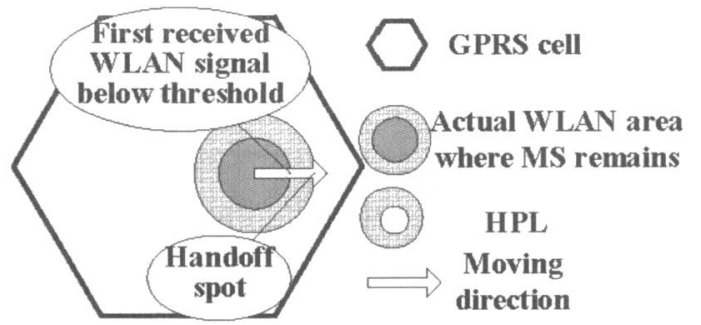

figure $2 \mathrm{HPL}$ : from WLAN to GPRS

The size of the loop is dynamic under different conditions according to the adaptive handoff decision algorithm[2]. For example, when MS moves slowly in GPRS cell towards WLAN area and the traffic of WLAN is low, algorithm in [2] encourages MS to switch to WLAN network, so the distance between the start point and the end point is short. When MS' speed is fast and the WLAN is busy, handoff to WLAN is discouraged, so the loop is wide. Low handoff latency and rapid signal exchanging is crucial for high speed MS. The HPL could provide more time for high speed MS to prepare for handoff, reducing outage probability consequently.

\section{B. WLAN-GPRS bi-direction connection}

Networks integration by Mobile IP will increase handoff latency. However, if Layer 3 connections with both GPRS and WLAN networks are established before Layer 2 handoff, namely bi-direction connection, outage will be reduced. Figure 3 shows the process.

$\mathrm{S}_{\mathrm{G}}$ : It is assumed that MS is in GPRS cell initially.

- $\mathrm{H}_{\mathrm{G1}}$ : New access is monitored. When new link is available, MS and systems could judge which network is currently serving. And Care-of Address is achieved.

- $\mathrm{H}_{\mathrm{G} 2}$ : When HPL is triggered, handoff signaling exchanges in the loop.

- $\mathrm{H}_{3}$ : Bi-direction connection registration is done if available resource allocation is finished.

- $\mathrm{H}_{\mathrm{W}_{4}}$ : Link layer handoff happens if the condition is met.

$\mathrm{H}_{5}$ :After binding update, handoff is finished.

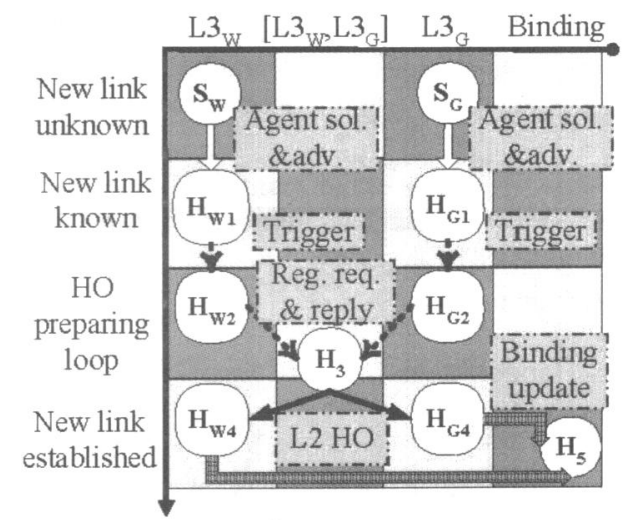

figure 3 WLAN - GPRS bi-direction connection

Although bi-direction connection could reduce handoff latency, it would occupy more network resources, especially for those users who are static in the HPL area. In this case, the bi-direction will maintain for ever unless the MS moves away or is turned off. So a parameter - bidirection Connecting Lifetime (CL) is introduced in order to solve the problem. The CL is inserted in the registration signaling to define the session of bi-direction connection.

\section{DETAILED handofF SIGNALING PROCESS AND} ANALYSIS

\section{A. Signaling flow}

In this section, detailed vertical handoff signaling process is presented according to previous description. Handoff trigger could be divided into two categories: network trigger and MS trigger. And the network trigger consists of two cases: the source trigger and the target trigger[6]. Only signaling with target trigger is shown in the paper for the limited space. Here the target means GPRS BS or WLAN AP. Signaling process for handoff to WLAN from GPRS is shown in figure 4. MN's IP address (Mobile Note's) in figure 4 is the Home Address, and FA's 
IP address is the Care-of Address. According to [7], MS could judge the new available access is WLAN or GPRS through Agent Advertisement and Solicitation messages, and achieve CoAs. After target trigger (start point of HPL), MS enters the HPL, and FA registers to HA. HA checks the registration message and reserves resource for MS, and then sends the reply message, which is cached in FA. Then the bi-direction connection is set up. HPL is over when Layer 2 handoff occurs. The signaling frames are defined in [7]. The CL value is inserted in the registration request message.

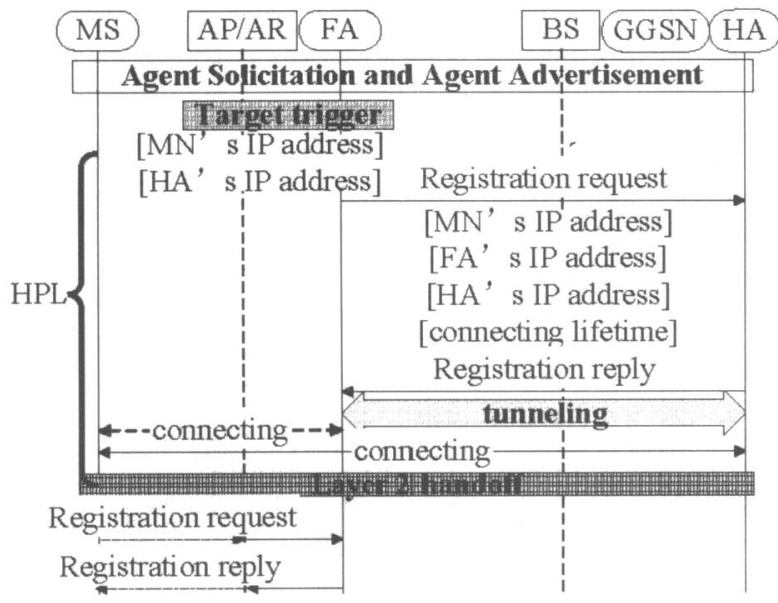

figure 4 Handoff signaling process : GPRS $\rightarrow$ WLAN

\section{B. Latency analysis}

Handoff delay basically consists of two components: link layer establishment delay and signaling delay. Link layer establishment is assumed to be negligible to signaling delay, so only signaling delay is accounted. Here define the handoff latency is the delay between registration messages sent and received by MS.

It is assumed that the delay between MS and the access network is tS. As shown in figure 4, the vertical handoff latency between GPRS and WLAN, namely tnho is defined as follows:

$$
\begin{aligned}
& t_{v h o}=\underbrace{t_{s}}_{\text {registration request to FA }}+\underbrace{t_{s}}_{\text {registration reply }} \\
& =2 t_{s}
\end{aligned}
$$

For the proposed signaling scheme, network layer handoff is prior to the link layer handoff; therefore the handoff latency and outage probability will be dramatically reduced. However, for the traditional Mobile IP signaling scheme[7], in which the link layer handoff occurs first and then the network layer handoff follows, tvho could be large enough to cause outage.

\section{Simulation Results}

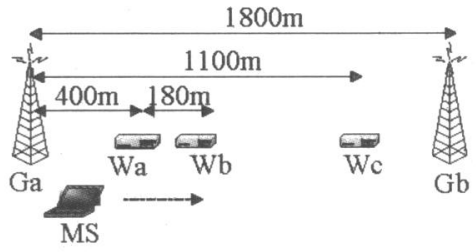

figure 5 simulation scenario

This section presents the simulation using MATLAB for $\mathrm{CL}$ and analysis of the results. The scenario for handoff is shown in figure 5. The parameters and algorithm have described in [2]. The MS moves from Ga to $\mathrm{Gb}$ at certain speed, and ongoing communication may handoff to $\mathrm{Wa}, \mathrm{Wb}$ or $\mathrm{Wc}$. Comparisons between systems with and without bi-direction connection are simulated, when CL takes different values, as shown in figure 6-10. Through comparisons the proper CL values are defined. The traffic of WLAN is 20 users in this simulation. MS's speed is $1,6,11,16$ and $21 \mathrm{~m} / \mathrm{s}$ respectively. The unit of $\mathrm{CL}$ is second, because the IETF Mobile IP defines that there is only one Agent Advertisement in one second at most, which means that handoff latency will not be shorter than one second.

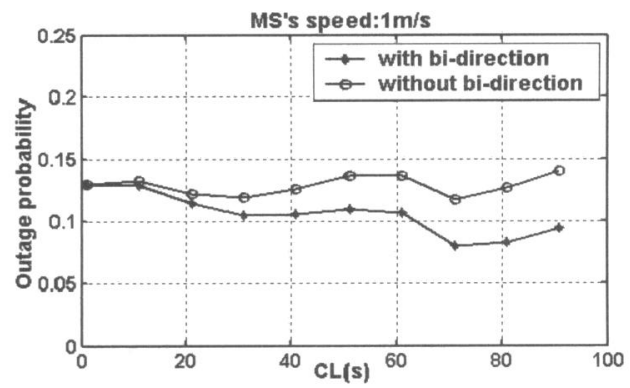

figure 6 Outage probability vs. CL when MS is slowest

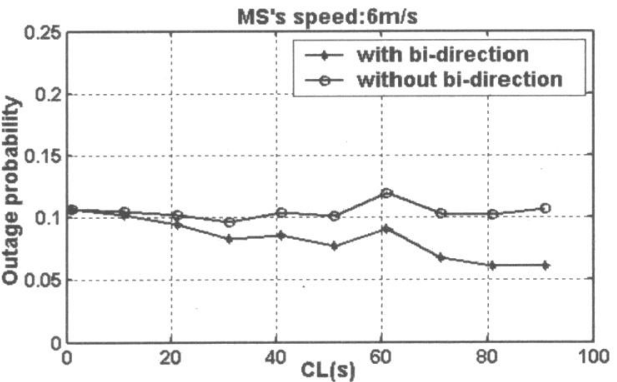

figure 7 Outage probability vs. CL when MS is slow 


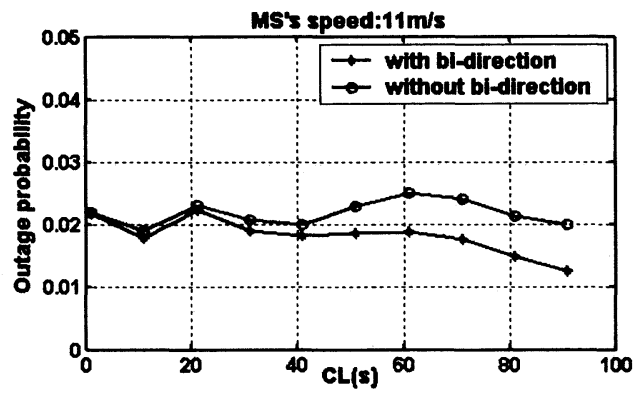

figure 8 Outage probability vs. CL when MS is normal

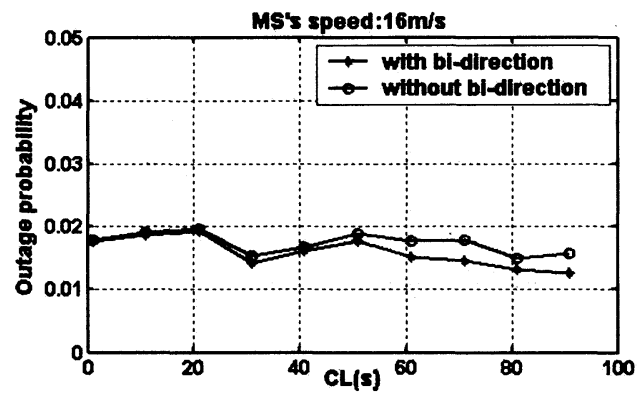

figure 9 Outage probability vs. CL when MS is fast

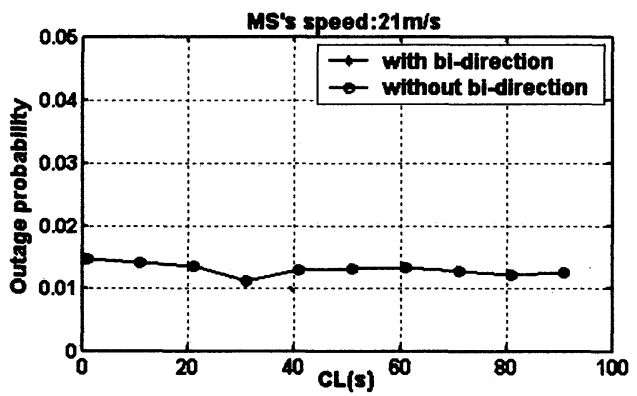

figure 10 Outage probability vs. CL when MS is fastest

Figure $6-9$ indicate that, for the system with bidirection connection, outage probability trends to decrease as $\mathrm{CL}$ becomes greater. However, with the increase of the velocity of MS, the disparity of outage probability at the same CL level is smaller and smaller. In figure 10, the disparity totally disappears due to the handoff algorithm discouraging vertical handoff when MS moves fast.

Table $1 \mathrm{CL}$ values

\begin{tabular}{|c|c|}
\hline States of MS's speed & Values of CL (s) \\
\hline Slowest & 10 \\
\hline Slow & 8 \\
\hline Normal & 6 \\
\hline Fast & 4 \\
\hline Fastest & 0 \\
\hline
\end{tabular}

Considering the tradeoff between outage probability and resource occupation, the value of $\mathrm{CL}$ is defined in table 1.The comparison between two systems under such
CL definition is shown in figure 11. Outage is reduced in the system with bi-direction connection when the velocity of MS is not fast.

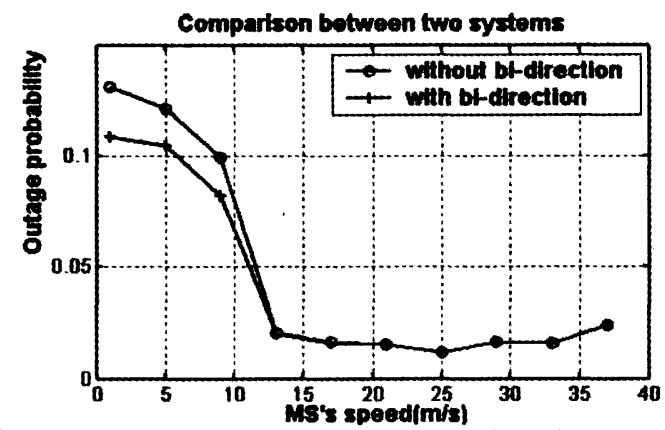

figure 11 Outage probability comparison between two systems

\section{Conclusion}

This paper proposes a signaling process scheme with QoS guarantee based on Mobile IP architecture for WLAN and GPRS networks. Vertical handoff latency is reduced through the design of Handoff Preparing Loop and Bi-direction connection. Detailed vertical handoff signaling process is described and analyzed. Simulation and analysis results indicate that the system with bidirection connection will achieve better performance in terms of outage probability and handoff latency.

\section{REFERENCES}

[1] T. S. Rappaport, "Wireless communication principle and practice, second edition", PHEI, 2004

[2] Q. Zhang, Y. H. Wang, et al., "Intelligent vertical handoffs between heterogeneous communication networks", accepted by The Journal of China Universities of Post and Telecommunications, (JCUPT), 2005

[3] A. Majlesi and B. H. Khalaj, "An adaptive fuzzy logic based handoff algorithm for interworking between WLANs and mobile networks," in Proc. IEEE PIMRC 2002, Lisbon, Portugal, vol. 5, Sep. 2002, pp. $2446-2451$

[4] I. F. Akyildiz, X. Jiang, and S. Mohanty, "A survey of mobility management in next-generation all-IP-based wireless systems" IEEE Wireless Communications, Vol. 11, No. 4, Aug. 2004 , pp. 16 - 28

[5] A. K. Salkintzis, C. Fors, and R. Pazhyannur, "WLAN-GPRS integration for next-generation mobile data networks," IEEE Wireless Communications, Vol. 9 , No. 5 , Oct. 2002, pp. 112 124

[6] K. E. Malki, "Low latency handoffs in mobile IPv4",draft-ietf-mobileip-lowlatency-handoffs-v409.txt, Dec. 2004

[7] C. E. Perkins, "IP mobility support for IPv4", IETF RFC 3344, Aug. 2002 